\title{
Certain Pure Cubic Fields With Class-Number One
}

\author{
By H. C. Williams
}

\begin{abstract}
A description is given of the results of some calculations performed to determine the class number of each of the pure cubic fields $Q(\sqrt[3]{q})$, where $q(\equiv-1(\bmod 3))$ is a prime and $q<35,100$. The stability of the percentage of these fields having classnumber one is examined.
\end{abstract}

Let $q$ be any prime such that $q \equiv-1(\bmod 3)$. In his review of [1] Shanks [7] noted that for primes $<10,000$, the fraction of the pure cubic fields $Q(\sqrt[3]{q})$ with classnumber one tended to remain about $48 \%$. In this note we present some results obtained from evaluating the class number $h$ of $Q(\sqrt[3]{q})$ for each $q<35,100$. The calculations were performed by using the methods of [1, Section 5]. Our main purpose is to study the constancy of this "about 48\%", since it remains unknown whether or not infinitely many algebraic number fields have $h=1$.

In Table 1 we give the values of $q$, the regulator $R(q)$ of $Q(\sqrt[3]{q})$, and $J$ the length of Voronoi's algorithm period for $\sqrt[3]{q}$, such that

$$
R(q)>R(r)
$$

for all primes $r(r \equiv-1(3))$ such that $8429 \leqslant r<q$.

\section{TABLE 1}

\begin{tabular}{ccc|ccc}
$q$ & $\mathrm{R}(\mathrm{q})$ & $\mathrm{J}$ & $\mathrm{q}$ & $\mathrm{R}(\mathrm{q})$ & $\mathrm{J}$ \\
10037 & 17941.60487 & 15972 & 21839 & 47361.35191 & 42122 \\
10067 & 18150.81288 & 16318 & 22469 & 47942.75017 & 42716 \\
11621 & 25661.99636 & 22908 & 26417 & 56816.82041 & 50385 \\
14897 & 28630.01878 & 25280 & 28517 & 57091.82492 & 50671 \\
15527 & 31541.56340 & 27991 & 29063 & 63398.84106 & 56707 \\
17669 & 32388.80366 & 28517 & 32213 & 71481.68242 & 63674 \\
19391 & 42811.86808 & 38337 & 34607 & 75693.99813 & 66931 \\
\hline
\end{tabular}

Since so many of the fields have $h=1$, the regulators are becoming very large; and consequently, the length of time the computer needs to evaluate them is also greatly increasing. It is because of the very large amount of time that the machine was spending in evaluating the regulators that the calculations were suspended when $q>35,100$.

In Table 2 we give for the 1880 primes $q<35,100$, each class number $h$ that occurs, the frequency $f(h)$ with which this $h$ occurs, the value of $100 f(h) / 1880$, and the smallest value of $q$ such that $h$ is the class number for $Q(\sqrt[3]{q})$, when this $h$ does not occur in Table 1 of [1].

Received July 9, 1976; revised July 29, 1976.

AMS (MOS) subject classifications (1970). Primary 12A50, 12A30, 12-04. 
TABLE 2

\begin{tabular}{rrrr|rrrr}
$\mathbf{h}$ & $\mathbf{f}(\mathbf{h})$ & Percentage & $\mathbf{q}$ & $\mathbf{h}$ & $\mathbf{f}(\mathbf{h})$ & Percentage & $\mathbf{q}$ \\
1 & 890 & 47.34 & & 56 & 2 & 0.11 & \\
2 & 486 & 25.85 & & 64 & 1 & 0.05 & \\
4 & 186 & 9.89 & & 68 & 1 & 0.05 & \\
5 & 49 & 2.61 & & 70 & 2 & 0.11 & \\
7 & 39 & 2.07 & & 71 & 3 & 0.16 & \\
8 & 72 & 3.83 & & 74 & 1 & 0.05 & \\
10 & 17 & 0.90 & & 80 & 1 & 0.05 & \\
11 & 10 & 0.53 & & 92 & 1 & 0.05 & 15131 \\
13 & 3 & 0.16 & & 95 & 1 & 0.05 & 15795 \\
14 & 11 & 0.59 & & 100 & 1 & 0.05 & 31547 \\
16 & 28 & 1.49 & & 104 & 2 & 0.11 & 11549 \\
19 & 1 & 0.05 & & 110 & 1 & 0.05 & 17333 \\
20 & 5 & 0.27 & & 127 & 1 & 0.05 & \\
22 & 6 & 0.32 & & 128 & 1 & 0.05 & \\
23 & 1 & 0.05 & 33149 & 136 & 2 & 0.11 & \\
25 & 2 & 0.11 & 10181 & 154 & 1 & 0.05 & \\
26 & 2 & 0.11 & & 175 & 1 & 0.05 & \\
28 & 12 & 0.64 & & 181 & 1 & 0.05 & 12251 \\
29 & 2 & 0.11 & 13331 & 200 & 1 & 0.05 & 12197 \\
31 & 1 & 0.05 & 16553 & 214 & 1 & 0.05 & 16823 \\
32 & 4 & 0.21 & & 230 & 1 & 0.05 & \\
34 & 2 & 0.11 & & 262 & 1 & 0.05 & 28979 \\
37 & 3 & 0.16 & & 284 & 1 & 0.05 & 24137 \\
40 & 4 & 0.21 & & 340 & 1 & 0.05 & 18257 \\
41 & 1 & 0.05 & & 358 & 1 & 0.05 & 27329 \\
44 & 4 & 0.21 & & 389 & 1 & 0.05 & 24023 \\
49 & 1 & 0.05 & & 748 & 1 & 0.05 & 17573 \\
50 & 1 & 0.05 & 22259 & 920 & 1 & 0.05 & 17579 \\
52 & 2 & 0.11 & & 1442 & 1 & 0.05 & 32771 \\
\hline
\end{tabular}

Let $n(x)$ be the number of primes $q(q \equiv-1(\bmod 3))$ which are less than or equal to $x$, and let $g(x)$ be the number of those primes such that the class number of $Q(\sqrt[3]{q})$ is one. For $x=1000,2000, \ldots, 35000$, we give in Table 3 the value of $100 g(x) / n(x)$.

TABLE 3

\begin{tabular}{cc|cc|cc}
$\mathbf{x}$ & $100 \mathrm{~g}(\mathrm{x}) / \mathrm{n}(\mathrm{x})$ & $\mathbf{x}$ & $100 \mathrm{~g}(\mathbf{x}) / \mathbf{n}(\mathbf{x})$ & $\mathbf{x}$ & $100 \mathrm{~g}(\mathbf{x}) / \mathbf{n}(\mathbf{x})$ \\
1000 & 51.72 & 13000 & 47.69 & 25000 & 47.37 \\
2000 & 49.35 & 14000 & 47.90 & 26000 & 47.42 \\
3000 & 47.30 & 15000 & 48.31 & 27000 & 47.56 \\
4000 & 46.76 & 16000 & 48.28 & 28000 & 47.50 \\
5000 & 48.82 & 17000 & 48.23 & 29000 & 47.60 \\
6000 & 48.74 & 18000 & 47.69 & 30000 & 47.70 \\
7000 & 49.01 & 19000 & 48.11 & 31000 & 47.50 \\
8000 & 49.51 & 20000 & 47.76 & 32000 & 47.68 \\
9000 & 48.40 & 21000 & 47.68 & 33000 & 47.33 \\
10000 & 47.65 & 22000 & 47.09 & 34000 & 47.46 \\
11000 & 47.55 & 23000 & 47.29 & 35000 & 47.31 \\
12000 & 47.11 & 24000 & 47.69 & & \\
\hline
\end{tabular}

The class number for each of the first 5000 fields $Q(\sqrt{p})$, where $p \equiv 1(\bmod 4)$ has been obtained by Kloss, Newman, and Ordman (see [6]). In [6] Shanks presented a table giving the number of values of $p$ in intervals of 1000 which have a particular 
class number. He noted that, for each successive group of 1000 of these primes, about $80 \%$ of the corresponding fields have class-number one. The apparent steadiness of this ratio prompted him to enquire as to whether it might be a fixed number as $p$ tends to infinity.

The data of Hendy [2] seem to indicate that the ratio of the number of $Q(\sqrt{d})$ ( $d$ is any square-free positive integer) with class-number one to the number of such fields with genus one is about $80.5 \%$. Hendy suggests that this ratio is $8 / \pi^{2}$ in the limit. Although the figure of $80 \%$ given above is for a somewhat different population than that considered by Hendy, he notes that the distributions of [6] and [2] are similar.

Lakein [3], [4] has obtained class numbers for 10000 quartic fields $K_{1}=F_{1}(\sqrt{\pi})$, where $F_{1}=Q(i)$ and $\pi=1+4 \alpha$ is a prime in $F_{1}$, and for 10000 quartic fields $K_{3}=$ $F_{3}(\sqrt{\pi})$, where $F_{3}=Q(\rho),(\rho=1 / 2(-1+\sqrt{-3}))$, and $\pi=a+b \rho$ is a prime in $F_{3}$ such that $a \equiv 1(\bmod 4), 4 \mid b, b>0$. The distributions he obtains for the number of each type of field having a particular class number are very similar to that of [6]. He also gives in [4] a distribution for $Q(\sqrt{p})(p \equiv 1(\bmod 4))$ obtained from previous results of Kuroda (see [5]). Kuroda extended the computations of Kloss, Newman, and Ordman to 100811 values of $p$. The percentage of these fields with class-number one is 77.65. This seems to indicate that this percentage is slowly decreasing as the number of values of $p$ increases.

On examining Table 3, it appears as if the value of $100 g(x) / n(x)$ is tending to stay between 47 and 48; however, with $x$ so limited it is impossible to say whether this trend will continue. The analogous ratios for the real quadratic fields and the special quartic fields mentioned above also seems to be stable within the limits of the tables presented in [6], [3] and [4]. However, we have seen that when more data for the real quadratic fields became available, the corresponding ratio decreased. This suggests that perhaps the value of $100 \mathrm{~g}(x) / n(x)$ might also decrease as $x$ gets large.

Department of Computer Science

University of Manitoba

Winnipeg, Manitoba R3T 2N2, Canada

1. PIERRE BARRUCAND, H. C. WILLIAMS \& L. BANIUK, “A computational technique for determining the class number of a pure cubic field," Math. Comp., v. 30, 1976, pp. 312-323.

2. M. D. HENDY, "The distribution of ideal class numbers of real quadratic fields," Math. Comp., v. 29, 1975, pp. $1129-1134$.

3. R. B. LAKEIN, "Computation of the ideal class group of certain complex quartic fields," Math. Comp., v. 28, 1974, pp. 839-846. MR 51 \#10290.

4. R. B. LAKEIN, "Computation of the ideal class group of certain complex quartic fields. II," Math. Comp., v. 29, 1975, pp. 137-144.

5. R. B. LAKEIN, "Review of UMT File: Table of Class Numbers, $h(p)$ Greater Than 1, for Fields $Q(\sqrt{p}), p \equiv 1(\bmod 4)<2776817, "$ Math. Comp., v. 29, 1975, pp. 335-336.

6. DANIEL SHANKS, "Review of UMT File: Class Number of Primes of the Form 4n+1," Math. Comp., v. 23, 1969, pp. 213-214.

7. DANIEL SHANKS, "Review of UMT File: Table of Pure Cubic Fields $Q(\sqrt[3]{D})$ for $D<$ $10^{4}$," Math. Comp., v. 30, 1976, pp. 377-379. 